\title{
Supressão condicionada: Um modelo experimental para o estudo da ansiedade*
}

\section{Conditioned supression: An experimental model for the study of anxiety}

\author{
Denigés Maurel Regis Neto ${ }^{1,2,3}$, Roberto Alves Banaco ${ }^{1,3}$, Nicodemos Batista \\ Borges $^{1,3,4}$ e Denis Roberto Zamignani ${ }^{3}$
}

\begin{abstract}
[1] Pontifícia Universidade Católica de São Paulo (PUC-SP), Brasil | [2] Centro de Análise do Comportamento (CeAC), Brasil | [3] Núcleo Paradigma de Análise do Comportamento, Brasil | [4] Universidade São Judas Tadeu (USJT), Brasil | Título abreviado: Supressão condicionada | Endereço para correspondência: Roberto Alves Banaco. Rua Wanderley, 611. CEP: 05011-001. São Paulo, SP. | E-mail: deniges@gmail.com
\end{abstract}

Resumo: O presente artigo apresenta uma breve perspectiva analítico-comportamental das emoções, a qual justificará a necessidade de estudos na área de supressão condicionada. Posteriormente, apresentar-se-á o modelo experimental da supressão condicionada, enfatizando os arranjos experimentais e descrevendo algumas variáveis envolvidas no fenômeno, relacionadas tanto ao condicionamento reflexo (S-S) quanto ao condicionamento operante (R-S) e que participam da produção de dados com sujeitos não humanos. Em seguida, serão apresentadas as pesquisas com participantes humanos que, utilizando os parâmetros das pesquisas descritas até então, tiveram como objetivo a produção de supressão condicionada, dadas as peculiaridades e os desafios particulares do tipo de participante. Para encerrar o artigo, apresentar-se-á a discussão sobre os pontos que necessitam de aprimoramento e desenvolvimento, visando a evocar nos leitores comportamentos de engajamento nesta linha de pesquisa.

Palavras-chave: supressão condicionada, interação operante-respondente, emoções

Abstract: This article presents a short behavioral-analytic view about emotions, justifying the need of studies about conditioned suppression, as an anxiety model. After, the conditioned suppression model is showed focusing the experimental analogs, and describing some of the major variables involved with this phenomenon, as those related to the reflex behavior (S_S relations) as those related to the operant relations (R-S relations) programmed to produce data in non-human behavior. Another focus of the article is over research with humans that, using procedures found in the literature had as their goals producing conditioned suppression, observing the peculiarities and the challenges due to this kind of participants. Finishing, a discussion is presented over aspects of this kind of research that claim for enhancing and development, aiming to invite the students and professionals to evolve this type of research.

Keywords: conditioned suppression, operant-respondent interaction, emotions

* Este texto foi produzido a partir da Introdução da dissertação de mestrado do primeiro autor, orientada pelo segundo autor no Programa de Estudos Pós-Graduados em Psicologia Experimental: Análise do Comportamento da Pontifícia Universidade Católica de São Paulo. 
Emoções têm sido um tema básico na psicologia. Vários autores, partindo de pressupostos científicos, buscaram interpretá-las por meio de processos respondentes e/ou fisiológicos (e.g., Watson, 1924).

No entanto, conforme Skinner (1953/2000) salientou, as diversas emoções, quando compreendidas apenas como reações fisiológicas, revelam poucas distinções entre si e têm limitada utilidade prática. Frequentemente, emoções são inferidas a partir da observação de um ou de alguns comportamentos em situações mais ou menos bem definidas e classificadas em relação a várias circunstâncias (variáveis) que afetam a sua probabilidade. Segundo Skinner, as variáveis referidas não devem ser atribuídas a condições psíquicas ou fisiológicas, mas sim a eventos ambientais, denominados naquela ocasião operações emocionais. "Definimos uma emoção, na medida em que se quer fazê-lo, como um estado particular de alta ou baixa frequência de uma ou mais respostas induzidas por qualquer uma dentre uma classe de operações" (Skinner, 1953/2000, p. 182).

O estudo das emoções a partir desta perspectiva implica a investigação das condições que compuseram as operações emocionais ocorridas na história do indivíduo e das condições atuais do ambiente que eliciam as ações respondentes ou evocam as mudanças no fluxo operante. Diz Skinner (1953/2000):

\begin{abstract}
Não é de qualquer auxílio, na solução de um problema prático, dizer-se que algum aspecto do comportamento do homem se deve à frustração ou à ansiedade; precisamos também saber como a frustração ou a ansiedade foi induzida e como pode ser alterada. (p. 184)
\end{abstract}

Skinner (1953/2000) destacou a importância das mudanças momentâneas no repertório geral de um indivíduo num "estado de emoção" (neste caso, medo ou fobia) com o seguinte exemplo:

Um homem de comportamento normal, em outras condições, pode revelar excessivo medo de pássaros mortos, por exemplo. ... provavelmente, poderíamos mostrar que a visão inesperada de um pássaro morto elicia respostas reflexas consideráveis - palidez, suor, mudanças nas pulsações executadas pela musculatura da face e do corpo. Se essa fosse a dimensão da fobia, poderíamos descrevê-la completamente como um conjunto de reflexos condicionados pela visão do pássaro morto, mas há outros efeitos importantes. O comportamento de fuga será bastante poderoso .... O restante do repertório passa por uma mudança geral ... se estiver empenhando em alguma outra ação, observamos uma alteração que pode ser descrita como "perda de interesse" .... Será menos provável que fale com a frequência natural; que ria, brinque e assim por diante ... essas mudanças podem persistir por um considerável período de tempo depois de o estímulo ter sido removido. Uma descrição completa da fobia precisa referir a todas elas [as mudanças observadas] e isto obviamente requer uma descrição do repertório comportamental inteiro do indivíduo. (pp. 183-184)

No trecho apresentado, Skinner explica as emoções a partir de uma interação respondente-operante. É justamente o estudo dos efeitos de condicionamentos respondentes sobre desempenhos operantes que define a linha de pesquisa a ser apresentada no presente artigo, conhecida como supressão condicionada.

Apresentaremos a seguir o modelo experimental da supressão condicionada, dando ênfase aos arranjos experimentais e descrevendo algumas variáveis envolvidas no fenômeno, relacionadas tanto ao condicionamento reflexo (S-S) quanto ao condicionamento operante (R-S) e que participam da produção de dados com sujeitos não humanos. Em seguida, apresentaremos as pesquisas com participantes humanos que, utilizando os parâmetros das pesquisas descritas até então, tiveram como objetivo a produção de supressão condicionada, dadas as peculiaridades e os desafios particulares do tipo de participante.

\section{A supressão condicionada enquanto um modelo experimental para o estudo da ansiedade}

Alguns fenômenos podem ser descritos quando se consideram os estímulos presentes no momento da alteração do repertório. Entretanto, outras situações tidas como emocionais parecem descrever 
perturbações provocadas por eventos futuros, o que induz a uma explicação antecipatória, teleológica. Este é o caso do estado de ansiedade. No senso comum, a ansiedade é vista como uma "expectativa" de que algo acontecerá. Ou, nas palavras de Estes e Skinner (1941), a ansiedade costuma ser descrita como uma "antecipação de um estímulo perturbador futuro" (p. 390).

Considerando que um estímulo que ainda não surgiu não poderia afetar uma resposta, Estes e Skinner (1941) salientaram a importância de identificar estímulos presentes que pudessem atuar como variável produtora dos efeitos descritos como ansiedade. Para tanto, consideraram que o paradigma do condicionamento reflexo forneceria elementos importantes para a discussão desse efeito.

No condicionamento reflexo, um estímulo eliciador incondicional de uma resposta é sistematicamente precedido pela apresentação de um estímulo inicialmente neutro para essa resposta. Em virtude dessa operação, o estímulo originalmente neutro adquire propriedades eliciadoras de respostas que preparam o organismo para o surgimento do estímulo eliciador incondicional ${ }^{1}$. O estímulo, antes neutro, passa a ser chamado de estímulo condicional (que precisou de uma condição - a operação de pareamentos - para adquirir a nova função eliciadora). As respostas eliciadas por esse estímulo condicional são chamadas de respostas condicionais. A extensão do controle exercido pelo estímulo incondicional (que ainda não ocorreu) sobre a resposta para o estímulo neutro (que se tornará, por pareamento, um estímulo condicional), presente no momento em que a resposta é observada, responderia ao problema da explicação teleológica.

\footnotetext{
1 A resposta condicionada, eliciada pelo estímulo condicional, pode ser similar à resposta incondicionada eliciada pelo estímulo incondicional, como no clássico exemplo do condicionamento da salivação canina de Pavlov. No entanto, em alguns casos, a reposta condicionada pode ser antagônica à resposta incondicionada. O efeito do condicionamento pode ser compreendido, então, como o de preparar o organismo para o estímulo que vem a seguir, condicionando respostas preparatórias, isto é, respostas adaptativas que mantenham o organismo homeostático, em equilíbrio.
}

\section{papel do condicionamento reflexo na} adaptação dos organismos

O condicionamento reflexo colabora para a adaptação do organismo, fazendo-o agir previamente ao estímulo incondicional que será apresentado, ou seja, prepara o organismo para a apresentação do estímulo eliciador incondicional. Por exemplo, imagine a vantagem adaptativa de um organismo que passe a salivar diante da mera apresentação de um limão (estímulo neutro que se tornará condicional). O sumo do limão em contato com a língua produz uma intensa resposta de salivação, atenuando os efeitos ácidos dessa substância. Dessa maneira, sumo de limão pode ser considerado um estímulo incondicional para a resposta reflexa incondicional de salivar abundantemente.

O estímulo é considerado incondicional porque não precisa (in) de nenhuma condição especial (condicional) para apresentar seus efeitos sobre o organismo. No entanto, sumo de limão na língua sempre é precedido pela apresentação do limão. Dados os pareamentos seguidos entre a apresentação do limão e o sumo do limão em contato com a língua produzindo salivação, a mera visão do limão (e algumas vezes a mera expressão verbal "sumo de limão na língua") passa a controlar a salivação, antes mesmo de o sumo ácido entrar em contato com a língua. $\mathrm{O}$ organismo se prepara para o contato com o sumo do limão, diminuindo os danos. Esta relação de "antecipação" ocorre porque na história desse organismo o estímulo presente (visão do limão) antecipou sistematicamente o estímulo incondicional (sumo do limão), tornando-se um eliciador condicional.

O estudo de interações respondentes e operantes: A inauguração de uma linha de pesquisa experimental de fenômenos emocionais

Baseados nas considerações feitas até agora, Estes e Skinner (1941) elaboraram um estudo que investigava a interação do respondente agindo sobre o desempenho operante, inaugurando a linha de pesquisa sobre supressão condicionada. Nesse primeiro estudo, os autores sobrepuseram um pareamento respondente (estímulo-estímulo ou S-S), entre um tom e um choque, a um responder operante (pressão à barra que produzia alimento ou R-S) e 
mediram as variações posteriores deste responder operante na presença do tom.

No experimento, os autores submeteram 24 ratos, distribuídos em dois grupos de acordo com os níveis de privação (definidos por eles como alto e baixo drive), a sessões de seis horas de duração, nas quais respostas de pressão à barra eram mantidas por reforçamento periódico (FI $4 \mathrm{~min}$ ) com liberação de alimento. O condicionamento do estado de ansiedade foi produzido pela apresentação de um tom, duas vezes por hora, com duração três minutos, cujo fim coincidia com a apresentação de um choque elétrico pelas grades do chão da caixa experimental (a intensidade do choque não foi mencionada no artigo).

Estes e Skinner (1941) observaram que não se evidenciou qualquer alteração significativa no responder operante na presença do tom ou posteriormente a ele em suas primeiras apresentações - o que caracterizaria esse tom como um estímulo neutro para as respostas emocionais. Segundo eles, a fim de esclarecer qualquer mudança no responder em estudo, o período de tom foi ampliado para cinco minutos, passando a ocorrer apenas uma vez por hora.

Nessa nova configuração temporal, o primeiro efeito observado, a partir da segunda apresentação do pareamento, foi uma redução a $30 \%$ das respostas operantes emitidas durante o tom, se comparada à mesma fração da sessão experimental controle (sem a sobreposição do tom-choque). Ou seja, a apresentação do tom passou a suprimir o responder operante depois do primeiro pareamento. Este efeito foi denominado pelos autores de supressão condicionada. Neste caso, é importante ressaltar que durante o tom os reforçadores para as respostas operantes ainda poderiam ser produzidos, caso $\mathrm{o}$ animal pressionasse a barra.

Os autores também identificaram um responder mais acelerado após o choque do que o responder observado antes do tom. Essa aceleração foi chamada por eles de aumento compensatório: a curva de respostas operantes alcançava um patamar próximo ao que estaria caso não tivesse ocorrido a diminuição observada na taxa do operante durante o tom. No entanto, essa taxa aumentada de respostas pós-choque não produzia mais reforço. $\mathrm{O}$ esquema de intervalos prevê que com um mínimo de respostas o animal pode produzir o total de reforços programados. Assim, pausas no responder, quando muito pronunciadas, produzem também uma diminuição no total de reforçadores obtidos na sessão experimental.

Em seguida, os pesquisadores submeteram o responder à apresentação tom-choque durante $e x$ tinção operante e também submeteram o operante a uma contínua apresentação do tom sem a presença do choque (extinção respondente), demonstrando que o efeito de supressão condicionada pode ser desfeito sob algumas condições. Os animais voltaram a emitir maior número de respostas durante o tom e, consequentemente, voltaram a produzir reforçadores durante a extinção respondente.

Sobre os resultados advindos das manipulações descritas até aqui, Estes e Skinner (1941) concluíram que uma redução na força de uma resposta operante pode ser produzida por repetidas apresentações de um estímulo originalmente neutro (o tom) terminado em um estímulo aversivo (neste caso, o choque elétrico). A redução na força é evidenciada pela diminuição da frequência daquela resposta operante durante as sucessivas apresentações do estímulo que historicamente precedeu o choque, o qual adquire as suas propriedades eliciadoras (torna-se condicional), sendo esse efeito mais marcante do que o produzido por um choque inescapável e não sinalizado.

Delineada dessa maneira, a supressão condicionada envolve, então, um arranjo entre contingências operante e respondente, representando o operante a variável dependente para o estudo dos efeitos do pareamento entre os estímulos condicional e incondicional. Estes e Skinner (1941) ainda destacaram que "a magnitude da reação a S1 [tom] a qualquer momento durante sua presença pode depender das relações temporais prévias de S1 [tom] e S2 [choque]" (p. 390). Isto é, o arranjo entre os estímulos da contingência respondente é uma variável importante na produção do efeito experimental denominado supressão condicionada do responder operante. Os próprios autores descreveram efeitos mais marcantes sobre o responder operante com a mudança da duração e da frequência do tom na fase experimental. 
levantamento de variáveis relevantes para a produção do efeito de supressão condicionada

\section{As relações temporais entre estímulos.}

O trabalho de Stein, Sidman e Brady (1958) mostra o efeito da manipulação das durações do estímulo condicional (CS - sigla da expressão inglesa conditioned stimulus) e do intervalo entre suas apresentações sobre um responder operante, com o propósito de testar diretamente essas variáveis.

Para isso, Stein et al. utilizaram cinco ratos albinos com cerca de 90 dias de vida, treinados a pressionar uma barra tendo como reforço três segundos de acesso à água. Inicialmente, todos os animais receberam sete horas de treino em esquema de VI 60s. Em seguida, três deles foram submetidos a sessões diárias de duas horas, tendo 30 horas de treino em VI 120s e acesso livre à água por 15 minutos após cada sessão. Os outros dois sujeitos foram submetidos a sessões de três horas de duração em noites alternadas, tendo 12 horas de treino em esquema de VI 120s e 40 horas de treino em VI 180s, com acesso livre à água por uma hora na manhã seguinte à sessão experimental.

Para produzir o efeito de supressão sobre o responder operante, os autores utilizaram como estímulo incondicional (US - sigla da expressão inglesa unconditioned stimulus) um choque elétrico de 0,5 s de duração e $1.0 \mathrm{~mA}$ de intensidade, apresentado ao fim de um período de estimulação sonora, um clicker (CS). Para cada sujeito, os pesquisadores delinearam uma sequência de variações temporais para a duração do CS e dos intervalos entre os CSs, referidos também como períodos $\mathrm{ON}$ (quando o estímulo condicional esteve presente) e OFF (quando o estímulo condicional não esteve presente).

De maneira geral, podemos compreender o arranjo como um delineamento $\mathrm{ABA}$, sendo que para cada sujeito foi produzida uma condição temporal de estímulos que se manteve por no mínimo 40 horas de treino até a estabilização do responder durante CS (Condição A). Em seguida, alterou-se apenas um dos valores temporais estabelecidos: o tempo de presença de CS (ON) ou o tempo de ausência do estímulo (OFF), também por no mínimo 40 horas de treino (Condição B). A terceira condição foi uma reversão à primeira configuração temporal da presen- ça/ausência do estímulo (Condição A - Reversão).

Manipulações adicionais foram realizadas para cada sujeito após esse delineamento básico, algumas delas permitindo comparações entre outros pares temporais de CS e intervalos entre CSs.

Assim, para alguns sujeitos o tempo de ausência (OFF) de estímulo era mantido constante durante as três condições (ABA), enquanto o tempo de presença de estímulo $(\mathrm{ON})$ era ampliado ou diminuído na Condição B. Para outros sujeitos, o período de estímulo presente era mantido constante durante as três condições, sendo manipulado para mais ou para menos o tempo do período de estímulo ausente. As durações de CS e dos intervalos entre CSs variaram de $30 \mathrm{~s}$ a $3000 \mathrm{~s}$, com diversos valores intermediários para cada um dos sujeitos.

Essas manipulações permitiram não só a comparação do desempenho do mesmo sujeito em diferentes condições temporais de CS ON e OFF, mas também do efeito das durações temporais relativas sobre a supressão das respostas. A duração relativa do período $\mathrm{ON}$ e OFF era calculada pela divisão do tempo de duração do estímulo ON pelo tempo de duração do estímulo OFF. Um arranjo cuja duração do clicker presente (ON) fosse de dois minutos e a duração do estímulo ausente (OFF) fosse de sete minutos tinha duração relativa de 0,29. Em alguns casos, arranjos de mesmo valor relativo tinham valores temporais bastante diferentes: por exemplo, intervalos de $30 \mathrm{~s} \mathrm{ON} \mathrm{/} 120$ s OFF têm a duração relativa igual à do período $240 \mathrm{~s} \mathrm{ON} / 960 \mathrm{~s}$ OFF, ambos com valor de 0,25.

A taxa de supressão era calculada pela taxa de respostas durante o período de CS ON dividida pela taxa de respostas no período de CS OFF, de mesma duração e imediatamente anterior à apresentação do CS. Nos casos de períodos de CS OFF mais curtos que de CS ON, a taxa utilizada seria a média das taxas da totalidade dos períodos $\mathrm{OFF}^{2}$.

2 Calculada dessa maneira, valores próximos a 1,00 indicam pouca supressão devido à reduzida diferença nas taxas de respostas no período de CS $(\mathrm{ON})$ e no período sem CS (OFF); valores próximos de 0,00 indicam grande supressão, pois resultam de uma diferença maior entre as taxas, sendo a do período de CS $(\mathrm{ON})$ menor do que a anterior à CS (OFF). Assim, relatar uma grande supressão é referir a valores próximos a 0,00 e relatar pouca supressão é referir a valores próximos a 1,00 . 
Os resultados apontaram que a supressão dependia da duração do período de CS ON em relação à duração dos períodos de CS OFF, e não da duração absoluta do período de CS: uma maior supressão ocorreu quando as durações dos CSs eram curtas relativamente aos períodos OFF. A taxa de supressão, portanto, seria inversamente proporcional à duração relativa do CS. Uma correlação de $90 \%$ foi encontrada entre a taxa de supressão e o logaritmo dos tempos relativos de CS ON e OFF.

Stein et al. (1958) notaram também que, independentemente da grande diferença nas taxas de supressão, os sujeitos obtiveram, em média, $90 \%$ do máximo de reforços possíveis na sessão. Isso significa que mesmo os arranjos temporais que mostraram grande supressão não implicaram a perda elevada de reforçadores (a perda máxima foi de apenas $10 \%$, bastante semelhante às perdas observadas nos arranjos produtores de supressões menores). Dessa forma, os autores sugeriram que as diferenças produzidas nas taxas de supressão poderiam estar relacionadas aos totais de reforços perdidos com a diminuição das respostas na supressão, e não apenas à configuração temporal dos estímulos envolvidos nas operações respondentes.

Os dados indicavam que supressões maiores ocorriam em arranjos nos quais a supressão no responder não implicaria grande perda de reforçadores (quando os períodos de CS eram relativamente curtos), enquanto supressões menores eram produzidas nos arranjos temporais cuja supressão implicaria perda acentuada de reforços (em períodos de CS relativamente longos). Novamente, uma alta correlação (92\%) foi encontrada, agora entre a taxa de supressão e a porcentagem estimada de reforços perdidos, caso houvesse total supressão do operante no período de CS.

Stein et al. destacaram, ainda, a correlação de 94\% entre os valores dos tempos relativos do CS (tempo de CS dividido pelo tempo sem CS) e a porcentagem da perda de reforço, caso houvesse total supressão. Observaram-se, então, três variáveis correlacionadas: a taxa de supressão (variável dependente), a duração relativa de CS e a quantidade de reforços perdidos em caso de supressão total (as duas últimas variáveis independentes). Todas essas variáveis correlacionaram-se em $90 \%$ ou mais entre si, o que dificulta a identificação de qual variável independente poderia ter um papel crítico na supressão condicionada.

\section{A frequência de reforçamento.}

Calton e Didamo (1960) apresentaram uma hipótese explicativa para os dados encontrados por Stein et al. (1958), a qual foi denominada minimização da perda de reforçamento. De acordo com Calton e Didamo, os sujeitos tenderiam a maximizar o acesso aos reforçadores ao longo da sessão experimental. Dessa maneira, em sessões de duração fixa, caso o sujeito apresentasse supressão pronunciada das respostas quando submetido a períodos relativamente grandes de CS, o baixo desempenho implicaria uma grande perda de reforços. Em função disso, os sujeitos tenderiam a manter a frequência do responder nessa condição, a despeito da apresentação do CS. Essa hipótese, de acordo com os autores, pressupõe uma sensibilidade (discriminação, segundo eles) dos sujeitos em relação à quantidade de reforços obtidos durante as sessões experimentais. A questão perseguida pelos autores foi: seria essa sensibilidade referente ao total de reforços obtidos numa sessão ou a uma fração momentânea da sessão (i.e., a uma taxa de reforçamento local)?

Para investigar essa questão, Calton e Didamo propuseram um arranjo experimental no qual o total de reforços obtidos fosse mantido constante em todas as sessões, independentemente do grau de supressão nos períodos de CS. Para isso, eles estenderam o tempo das sessões até que o total de reforços estipulado fosse atingido. Assim, os sujeitos poderiam obter todos os reforçadores possíveis em uma sessão, o que possibilitaria a supressão das respostas mesmo em CSs relativamente mais longos. Dessa forma, seria possível verificar se a taxa de supressão produzida era efeito do total de reforços obtidos/perdidos na sessão ou da duração relativa de CS. Segundo a hipótese dos autores, o responder deveria apresentar supressão mesmo nos períodos longos de CS, uma vez que isso não implicaria a diminuição do total de reforços obtidos na sessão. Por outro lado, caso os dados encontrados fossem semelhantes aos de Stein et al. (1958), isso indicaria que os sujeitos eram sensíveis apenas a uma frequência de reforçamento disponível momentaneamente (taxa local de reforçamento). 
Calton e Didamo (1960) utilizaram dois ratos albinos mantidos a $65 \%$ do peso ad lib, respondendo com pressões à barra pela obtenção de 0,1 $\mathrm{cm}^{3}$ de uma solução de leite condensado diluído em água, em um esquema de VI 120s. O estímulo condicional era um tom apresentado por $180 \mathrm{~s}$, o estímulo incondicional era um choque de $0,5 \mathrm{~s}$ de duração e 1,5 a $2 \mathrm{~mA}$ de intensidade. Na primeira fase, o tempo da sessão era fixo, de três horas, nas quais a apresentação do tom ocorria uma vez por hora. Na segunda e terceira fases, o tempo da sessão era estendido até que o número total de reforços programado fosse obtido. Este número de reforços foi estabelecido a partir da média de reforços das últimas três sessões da primeira fase. A duração do período sem tom (intervalo entre tons) foi diminuída de 57 minutos para três e um minutos na segunda e na terceira fase, respectivamente, enquanto o período de tom foi mantido constante em três minutos em todas as fases.

Os dados foram apresentados na forma de taxa de supressão, ou seja, divisão da taxa de respostas no período de tom pela taxa no período sem o tom, referente às últimas cinco sessões das 25 de cada fase. Assim como descrito em Stein et al. (1958), os valores menores na razão obtida nesse estudo indicariam maior supressão, enquanto valores maiores indicariam pouca supressão.

Calton e Didamo (1960) verificaram que a supressão foi menor em cada fase sucessiva do experimento. Tal resultado é compatível com o obtido por Stein et al. (1958), ocorrendo menor supressão em períodos relativos de CS mais longos, independentemente da quantidade de reforços obtidos na sessão. Não tendo encontrado diferenças na taxa de supressão nas várias durações do CS utilizadas ao manter o total de reforços obtidos, Calton e Didamo assumiram que a variável relevante na produção de supressão condicionada seria o total de reforços obtidos em um período mais curto (taxa local de reforçamento) - o que, por sua vez, dependeria da frequência de reforçamento no esquema operante vigente.

Procurando aprofundar o conhecimento sobre as relações entre as variáveis já estudadas, Lyon (1963) produziu dados que permitiram verificar os efeitos das diferentes condições temporais de CS, quando sobrepostas a diferentes frequências de reforçamento durante a apresentação do CS. Para investigar o efeito do pareamento sobre o desempenho em esquemas de diferentes frequências de reforçamento, Lyon utilizou dois pombos mantidos a $70 \%$ do peso ad lib e submetidos a sessões de 110 minutos de duração, em esquema múltiplo de VI 60s e VI 240s, associados respectivamente à luz vermelha e à luz verde. Os esquemas componentes do múltiplo eram apresentados de forma alternada, cinco vezes por sessão, com duração de 10 minutos cada. A mudança de um componente para outro era intercalada por um período de escurecimento da caixa experimental durante um minuto, no qual as respostas de bicar o disco não produziam reforçamento. O US utilizado foi um choque de 90 volts de 0,02 s de duração pareado a um CS, representando por uma sequência de flashes produzidos pela iluminação da caixa (com frequência de 100 brilhos por minuto). A apresentação deste CS ocorria dois minutos após o início de cada componente do esquema múltiplo, totalizando 10 ocorrências por sessão, sendo cinco em cada componente.

O período de CS foi ampliado gradualmente, tendo inicialmente $100 \mathrm{~s}$ de duração e, após a estabilização da taxa de supressão, foi aumentado para 200 s. Uma vez obtida nova estabilidade, a duração foi ampliada para 300 s. O experimento utilizou como medida de supressão a divisão entre a frequência média de respostas por minuto na presença de CS e a frequência média de respostas na ausência de CS.

Os resultados encontrados por Lyon (1963) quanto ao efeito da variação temporal de CS sobre a taxa de supressão corroboram os dados de Stein et al. (1958) e de Calton e Didamo (1960), nos quais a supressão é inversamente proporcional à duração relativa do CS. Seus dados confirmaram também a expectativa de Calton e Didamo a respeito da diferença da taxa de supressão em função das diferentes frequências de reforçamento, pois a supressão foi maior no componente de menor frequência de reforçamento em todas as durações de CS, indicando uma menor perda de reforçadores, caso fosse observada a supressão.

Lyon (1963) também observou diferenças quanto ao aparecimento e à eliminação dos efeitos supressivos de CS perante as diferentes frequências de reforçamento. O efeito supressivo para o com- 
ponente de maior reforçamento foi observado de maneira mais atrasada do que para o componente de menor reforçamento. Posteriormente, com a apresentação do flash sem a apresentação do choque, observou-se uma recuperação mais rápida do responder durante CS no componente de maior frequência de reforçamento. Assim, para ambos os sujeitos e em todas as durações de CS, os esquemas com menor frequência de reforçamento apresentaram taxa de supressão mais expressiva e rápida, com maior durabilidade após a quebra do pareamento flash-choque.

Lyon (1963) alertou para que não se assumisse precipitadamente a frequência de reforçamento por si como uma variável determinante na supressão. O autor sugeriu que os diferentes graus de supressão poderiam estar relacionados, não apenas aos esquemas de reforço vigentes, mas também à frequência de respostas na ocasião em que o CS era apresentado, fato que o levou a propor novos experimentos.

\section{Os diferentes esquemas de reforçamento.}

Nessa linha de pesquisa, Lyon (1964) e Lyon e Felton (1966) produziram dados sobre a supressão condicionada em esquemas de razão fixa e variável com pombos. Em Lyon (1964), dois sujeitos submetidos a um esquema de razão fixa (FR 150) recebiam choques de 30 volts por $0,2 \mathrm{~s}$ ao final de um estímulo sonoro de um minuto apresentado em intervalos de três a sete minutos.

Os dados indicaram que, ao longo de uma cadeia de respostas em um esquema de razão, o momento de apresentação do estímulo sonoro (CS) era crucial para o grau de supressão do responder. Quando ele apresentado no início da cadeia (até a vigésima resposta), observava-se total supressão das respostas, a qual permanecia até o final da apresentação do estímulo. Quando o CS era apresentado entre a vigésima e a sexagésima respostas, uma supressão parcial era observada. Já a partir da sexagésima resposta, os sujeitos respondiam a despeito da apresentação do CS, até atingir a centésima quinquagésima resposta e obter o reforçador.

Por outro lado, quando submetidos a esquemas de razão variável (VR 50, 100 e 200), os três sujeitos de Lyon e Felton (1966) apresentaram muita variabilidade nos resultados. Apesar de demonstrarem alguma supressão durante a apresentação do CS, ela ocorria em valores bastante diferentes entre si (a taxa de supressão variou entre 0,4 e 0,8 ). Em algumas ocasiões, os sujeitos ainda responderam frequentemente durante o CS, enquanto em outras situações apresentaram total supressão do operante.

É digno de nota o fato de o efeito supressivo ter se manifestado de maneira bem distinta, mesmo sob condições de reforçamento e apresentação de CS e de US semelhantes. Os dados de Lyon (1964) e Lyon e Felton (1966) apontam para a importância não apenas da frequência do reforçamento, mas também do próprio esquema operante envolvido na produção dos reforçadores, como elementos determinantes da interação operante-respondente que resulta em supressão condicionada.

A partir desses estudos, destacam-se mais duas variáveis que podem agir sobre a produção da supressão perante um estímulo condicionado à estimulação aversiva: (a) as condições de produção de reforço, ou seja, os esquemas e as frequências de reforçamento e (b) o momento de apresentação do CS em relação à contingência de produção do reforço.

Em busca de mais variáveis envolvidas no fenômeno da supressão condicionada, Blackman (1968a) também identificou em esquema múltiplo DRL FI, ambos com retenção limitada da disponibilidade do reforço (limited hold), maior resistência à supressão durante CSs apresentados mais próximos ao momento do reforçamento no esquema de FI. Blackman (1977) reuniu esses e outros dados para destacar os efeitos da iminência do reforçamento sobre o grau de supressão: quanto mais iminente se encontrava o reforço, menor a supressão durante o CS.

Com base nesses estudos, cabe argumentar que as diferenças na relação entre as respostas e a produção do reforçamento nos distintos esquemas (de razão ou de intervalo, fixos ou variáveis) podem auxiliar na compreensão das diferenças e das peculiaridades da supressão encontrada quando cada um deles foi utilizado como base para o procedimento respondente ser superposto.

Nos esquemas de intervalo, uma resposta produzirá reforço desde que seja emitida após um determinado tempo mínimo a partir do último reforçamento liberado, independentemente do número de respostas emitidas durante esse intervalo. Os esquemas de razão implicam a emissão de um 
número determinado de respostas desde o último reforçamento para que um novo ocorra, independentemente do tempo requerido para isso. Por estas características da programação dos reforços, uma pausa no responder em cada um desses dois esquemas resultaria em efeitos muito diferentes: em ambos, uma pausa do responder implicará um atraso do reforçamento; porém, nos esquemas de razão, a probabilidade de reforçamento será igual antes e após a pausa do responder. Qualquer diminuição na frequência das respostas implicará um atraso do reforçamento equivalente a essa diminuição. Já nos esquemas de intervalo, a passagem do tempo, por si, torna mais provável o reforçamento da próxima resposta. Uma pausa do responder ou uma diminuição em sua frequência a níveis muito baixos (desde que não seja extrema o suficiente para alterarem os valores do esquema proposto) não redundará em uma diminuição na taxa de reforçamento equivalente à diminuição da taxa do responder, conforme já demonstraram Stein et al. (1958).

Pode-se supor, portanto, que a supressão das respostas operantes diante de um estímulo condicionado a um estímulo aversivo seja mais provável em esquemas de intervalo, pois, neste caso, tal supressão traria menor prejuízo na taxa de reforços produzidos do que quando ela acontece nos esquemas de razão. Pode-se ainda estender o raciocínio para as diferenças encontradas por Lyon (1963) na comparação das frequências de reforçamento (VI 60s e VI 240s). A iminência do reforçamento no caso do VI 60s sempre era consideravelmente maior do que no caso do VI 240s, o que poderia justificar a diferença nas taxas de supressão perante o CS. Assim, a iminência do reforçamento, talvez substituível por probabilidade local de reforço, pode ser uma variável crítica na compreensão da supressão condicionada.

Essas considerações podem ajudar a entender a irregularidade nos dados de supressão em esquemas de razão variável de Lyon e Felton (1966). Nesses, a iminência do reforço aumenta a cada resposta, mas tal aumento ocorre de maneira variável a cada nova sequência, o que poderia produzir as oscilações entre alta e baixa supressão. Isto é, as oscilações na supressão acompanhariam as oscilações entre alta e baixa probabilidade de reforçamento.
Tal hipótese é contestada, entretanto, pelos dados obtidos por Blackman (1977). O autor relatou brevemente que, em 1966, ao colocar sujeitos em VR 100 acoplados (yoked) a outros que trabalhavam em um esquema de VI, recebendo reforço apenas quando os primeiros também os produzissem, encontrou clara supressão no responder em VR e uma taxa de supressão menos pronunciada no responder em VI durante a apresentação do CS.

Portanto, em esquemas que envolvem uma codependência entre a passagem do tempo e a ocorrência de uma resposta (esquemas de intervalo), a literatura mostra que a sobreposição do pareamento entre um CS e um estímulo aversivo tem um efeito mais pronunciado de supressão do que quando sobreposto a esquemas que dependem exclusivamente da ocorrência de uma determinada quantidade de respostas (esquemas de razão), lembrando que dados assistemáticos aparecem quando os esquemas de razão são variáveis.

Algumas outras questões podem ser levantadas quanto à frequência de respostas envolvidas em cada um dos valores de VI. Pode-se pensar que a relação entre quantidade de respostas por reforçamento seja uma variável relevante na produção de supressão condicionada. Segundo Blackman (1977), altas frequências de respostas são mais passíveis de supressão do que baixas frequências. O autor apresenta uma divergência em relação ao trabalho de Lyon (1964), o qual sugere que as altas taxas de respostas encontradas no esquema de reforçamento mais frequente (e.g., VI 60s) sofreriam menor supressão. Para Blackman (1977), o arranjo de Lyon (1964) é problemático por não controlar as taxas de reforçamento por respostas entre os VIs 60s e 240s. O delineamento de Blackman (1968b) propiciou a manutenção das taxas de reforçamento por respostas em diferentes valores de esquemas, acrescentando condições de DRL e DRH aos VIs, as quais levavam a baixas e a altas taxas de respostas para a produção de reforçadores.

Pode-se ainda analisar as divergências nos dados pelas diferenças nos arranjos experimentais e em sua consequente relação com a quantidade de respostas por reforçamento. No arranjo de Lyon (1964), os diferentes valores de VI produziram diferentes frequências de respostas, mas não revelaram qual a relação entre a quantidade de respostas por 
reforço, dado que em ambas as condições poderiam ter sido semelhantes. $\mathrm{O}$ arranjo de Blackman (1968b) produziu, inevitavelmente, quantidades de respostas bastante diferentes para cada condição (VI com DRL e VI com DRH). Nelas, a diferença na supressão poderia ser atribuída às diferenças quanto à quantidade de respostas por reforço, e não às taxas de respostas por si. Os resultados de Blackman (1968b) podem ter contrariado os de Lyon (1963) devido à diferença entre o número de respostas por reforçamento, e não diretamente pelas taxas de respostas.

\section{Os efeitos de diferentes eventos aversivos como US}

Destacou-se até aqui as implicações dos esquemas de reforçamento e das durações relativas de CS para a produção de supressão condicionada.

Algumas outras questões relativas ao pareamento entre CS e US também são tratadas na literatura. Além dos estudos dos tempos relativos, investigações quanto ao tipo de evento aversivo que ocupa o lugar de US são importantes e revelam fatos relevantes para a continuidade das investigações e para considerações acerca de outros assuntos no controle aversivo.

Leitenberg (1966) manipulou o tipo de evento subsequente ao CS: choque elétrico, som alto e time out (suspensão da contingência de reforçamento) em dois experimentos.

No primeiro experimento, Leitenberg comparou os efeitos do choque elétrico e da utilização de time out (TO) com quatro pombos mantidos a $75 \%$ do peso ad lib, submetidos a esquema de VI 120 s e VI 180s com o disco iluminado por uma luz branca. Luzes vermelha ou verde no disco antecediam por 30 segundos o evento aversivo. A luz vermelha antecedia um choque elétrico de duração $40 \mathrm{mls}$ no púbis das aves, com diferentes intensidades para cada sujeito, não relatadas pelos pesquisadores. A luz verde antecedia um período de TO de 10 minutos, durante o qual a luz do disco era desligada. Essas duas cores de luz eram apresentadas 10 vezes por sessão, alternadamente, em intervalos de nove minutos.

Esse arranjo foi adaptado para estudar também a comparação entre os efeitos do choque e do TO produzidos pela resposta e não produzidos pela resposta. Quando em condição de dependência da resposta, as luzes verde e vermelha eram apresentadas até que o sujeito respondesse, produzindo assim o choque ou o TO.

$\mathrm{Na}$ condição de independência da resposta para a ocorrência do evento aversivo, foi observada uma elevação do número de respostas durante a luz verde, terminada em TO em três sujeitos.

No Experimento 2, Leitenberg (1966) propôs o mesmo arranjo das apresentações das luzes e de reforçamento, substituindo choque por um som alto, para três pombos sob as mesmas condições dos sujeitos do Experimento 1. Dessa vez, o TO era antecedido pela luz vermelha e o som alto pela luz verde, para comparar possíveis efeitos da cor sobre os eventos aversivos. Nenhuma fase de dependência ou independência da resposta foi descrita nesse experimento.

Os resultados mostraram novamente uma aceleração das respostas durante o estímulo que antecedeu o TO. A discussão feita pelo autor acrescenta que um estudo realizado por Pliskoff, em 1961, teria encontrado resultados que descrevem aumentos na frequência das respostas durante um estímulo que antecedia uma diminuição na frequência de reforçamento do esquema em vigor.

Deve-se destacar que, diferentemente da ideia clássica da supressão condicionada, o estudo de Leitenberg (1966) produziu uma alteração na contingência de reforço em vigor, suspendendo a contingência de reforçamento como parte do pareamento. Os arranjos que produzem supressão têm sido construídos com eventos paralelos à contingência de reforçamento - os quais, quando presentes, interferem na frequência das respostas operantes. Construindo uma condição na qual a contingência é suspensa, Leitenberg interferiu no elemento que torna o fenômeno da supressão condicionada interessante: o de uma condição paralela afetar uma contingência operante em vigor.

Como pode ser notado até aqui, a explicação dos fenômenos da supressão ainda é uma questão a ser considerada. Blackman (1977) apresenta três hipóteses, as quais envolvem: (a) punição acidental e ocasional do comportamento operante, (b) mudança de aspectos motivacionais do organismo e (c) interferência de outras respostas operantes e respondentes que afetariam a contingência operante medida. 
Outras questões ainda podem ser feitas quanto à influência da magnitude do reforçamento na supressão (Geller, 1960; Leslie, 1977) e dos estados de privação. Há ainda estudos - que não serão o foco do presente artigo - sobre as relações de generalização do efeito do CS (Hoffman \& Fleshler, 1961, 1964; Hoffman, Selekman, \& Fleshler, 1966; Ray \& Stein, 1959).

\section{estudo de supressão condicionada em humanos}

Um fato evidente a partir das pesquisas apresentadas é o de que poucas tentativas de estudo desse fenômeno foram realizadas com humanos.

Um estudo de supressão condicionada com humanos ocorreu como parte de um projeto que visa a desenvolver tecnologias capazes de medir e intervir sobre o fenômeno da "ansiedade". Banaco et al. (2004) realizaram uma pesquisa que tinha como objetivo construir um modelo análogo ao de Estes e Skinner (1941) para o estudo com seres humanos em situação experimental. Uma questão fundamental, levada em consideração na programação do análogo, era a escolha do estímulo aversivo a ser utilizado no estudo. Por questões éticas, os pesquisadores utilizaram estímulos de intensidade amenizada.

Para o estudo, os pesquisadores contaram com 22 participantes de ambos os gêneros e com idades entre 18 e 30 anos. Na primeira fase, o objetivo foi identificar uma palavra que pudesse ter algum efeito aversivo para cada um dos participantes. Para isso, palavras eram apresentadas em pares - selecionados aleatoriamente pelo programa de uma lista que continha 20 palavras consideradas positivas (e.g., felicidade e amor), 20 neutras (e.g., cadeira e computador) e 20 negativas (e.g., roubo e câncer) e o participante era orientado a clicar naquela que ele considerasse mais negativa. Ao clicar, aquelas palavras sumiam da tela e um novo par era apresentado. Esse procedimento se repetia até que todas as palavras fossem apresentadas com todas as outras, sendo a palavra mais clicada aquela escolhida para exercer função "aversiva" (para aquele participante) e substituir o choque utilizado na pesquisa de Estes e Skinner (1941). A escolha por palavras para exercerem função aversiva se baseou em estudos (e.g., Leslie, Tierney, Robinson, Keenan \& Watt, 1993;
Watt, Keenan, Barnes \& Cairns, 1991) que sugerem que palavras podem pertencer a classes de estímulos com funções aversivas. Outro fator que contribuiu para essa escolha foram as implicações éticas no uso de estímulos diferentes, tais como choque.

A segunda fase do estudo de Banaco et al. (2004) consistiu na construção da linha de base. Nela, os participantes eram orientados a clicar em letras de um teclado virtual, disposto na tela do computador sobre um fundo cinza, com o objetivo de formar palavras de cinco letras, simulando um Jogo de Forca. Clicar sobre o teclado virtual, portanto, era a resposta operante que estava em foco nesse estudo.

A construção da linha de base consistiu em consequenciar com pontos as respostas de clicar o mouse. Num primeiro momento, programou-se um esquema de reforçamento contínuo em que se ganhava um ponto - mostrado em um contador localizado à direita na tela do computador - a cada clique sobre uma letra correta e 10 pontos e um som dizendo "Parabéns!" a cada clique sobre a última letra que completava a palavra (as palavras eram escolhidas aleatoriamente pelo programa). $\mathrm{O}$ participante tinha 13 chances para acertar as cinco letras da palavra. Caso o participante não completasse a palavra nas 13 chances, era apresentado um som dizendo "Perdeu!" e se iniciava uma nova tentativa com outra palavra. Num segundo momento, o esquema de liberação de pontos passou a ser de razão variável (VR), mantidas todas as demais condições de programação.

A terceira fase tinha por objetivo verificar os possíveis efeitos da associação entre o pareamento da cor de fundo da tela com a apresentação da palavra escolhida como "mais aversiva" sobre o responder dos participantes (i.e., clicar o mouse). Para isso, as únicas alterações em relação à segunda fase foram: (a) de tempos em tempos, a intervalos variados e de modo não contingente ao responder do participante, o fundo da tela deixava de ser cinza e se tornava vermelho, permanecendo assim durante 60 segundos; (b) ao término dos 60 segundos do Jogo de Forca sobre fundo de tela vermelho, surgia na tela do computador a palavra mais aversiva eleita pelo participante na primeira fase. Após o seu aparecimento, a tela voltava a ter o fundo cinza. $\mathrm{O}$ Jogo de Forca funcionava durante todo o tempo do 
procedimento. Nessa fase, as contingências de liberação de pontos pelo clicar a letra certa ou terminar a palavra permaneceram as mesmas do final da segunda fase (i.e., razão variável).

Os resultados permitiram a identificação de três padrões de responder durante a apresentação da cor vermelha no fundo de tela na terceira fase: (a) cinco participantes não apresentaram alterações no padrão de responder; (b) quatro participantes apresentaram um padrão em que houve uma redução do responder logo após a mudança da cor do fundo da tela, com um aumento na frequência do clicar durante o minuto e uma elevação acentuada de respostas segundos antes da apresentação da palavra mais aversiva e (c) sete participantes apresentaram estabilidade no responder, havendo diminuição da frequência de respostas apenas por alguns segundos antes da apresentação da palavra mais aversiva.

Diante desses resultados, Banaco et al. (2004) concluíram que nenhum dos participantes apresentou um padrão tipicamente encontrado nos estudos de supressão condicionada com sujeitos não humanos. Contudo, eles ressaltaram que a mudança de cor do fundo da tela e/ou a manipulação da palavra eleita como mais aversiva exerceram influência sobre o responder de metade dos participantes.

Em sua discussão, Banaco et al. (2004) tecem algumas considerações acerca das três fases do experimento. Sobre a primeira fase, os autores indicam que a seleção do estímulo que exerceria a função de aversivo na primeira fase pode ter produzido resultados contraditórios, na medida em que o participante deveria escolher a palavra que mais rejeitava. No entanto, é possível que a instrução não tivesse ficado clara para o participante e ele pode ter clicado várias vezes sobre sua palavra preferida, e não sobre a palavra "mais rejeitada". O método de seleção da palavra poderia ter implicado que a palavra mais escolhida fosse utilizada como estímulo pareado à troca de cor do fundo de tela.

Sobre o desempenho em linha de base, sobre a qual se realizou o estudo, Banaco et al. (2004) discutem que esta envolveu diversas respostas de observação, de identificação de letras e de escolha, as quais não puderam ser medidas e que talvez tenham sido relevantes para os resultados. Os próprios esquemas de CRF e VR utilizados, se de fato os considerarmos análogos, produzem desempe- nhos problemáticos para a produção de supressão condicionada, considerando o que foi dito anteriormente no presente artigo. Outro problema é que uma palavra completa corretamente produziria 10 pontos. Assim, teríamos simultaneamente um esquema de VR para cada letra escolhida e uma VR variando de 5 a 13 que produziria 10 pontos. Ainda deve ser lembrado que os pontos não eram trocados por nenhum objeto tangível, o que também pode ser discutido. No entanto, o estudo foi baseado em Costa (2004) e Tomanari, Carvalho, Góes, Lira e Viana (2007), autores que demonstraram que humanos adultos ficam sob controle de ganho ou perda de pontos não trocáveis.

Quanto ao estímulo aversivo selecionado, pode-se reconhecê-lo também como um estímulo condicionado. É necessária uma longa história de pareamentos para que uma palavra escrita se torne um estímulo controlador de comportamento. A tentativa de pareamento realizada entre cor (CS) e palavra (também um CS, e não um US) seria, portanto, de segunda ordem, diferente dos estudos com não humanos que utilizaram estímulos aversivos incondicionados.

A duração relativa da tela vermelha parece ter sido também um problema, já que estabeleceu uma duração relativa alta (60 segundos), para a qual os estudos de Stein et al. (1958), Calton e Didamo (1960) e Lyon (1964) demonstraram produzir menor supressão.

Considerando essas observações, é necessário criar condições experimentais que permitam clareza e controle quanto ao esquema, à frequência e à magnitude do reforçamento, além de monitoramento cuidadoso sobre a duração de CS em relação à sessão. Ao mesmo tempo, é importante a escolha de um evento aversivo mensurável e manipulável quanto à magnitude.

Uma alternativa ao uso de estimulação aversiva por apresentação de reforçadores negativos pode ser a retirada de reforçadores positivos fornecidos pela própria condição experimental. A perda de reforçadores produzidos pelo participante seria uma alternativa. No entanto, Leitenberg (1966) demonstrou que a suspensão da condição de reforçamento (time out) produziu um aumento nas respostas durante a apresentação do CS. Pode-se esperar que um estímulo que anteceda a perda de reforçado- 
res, uma vez produzidos pelo responder, interfira no desempenho a ser manipulado, tornando esse responder mais frequente. Isso corresponderia a uma interferência na contingência de reforçamento. Uma alternativa seria a perda de algum reforçador não produzido pela resposta medida, que assim não faria parte da contingência em vigor. Levando-se em conta tais dados, a obtenção de reforçadores por esquemas de tempo (FT ou VT), contingente à permanência na sessão, seria uma opção melhor, pois exigiria apenas a resposta de permanência no experimento para a sua produção e a sua perda não interferiria diretamente na produção de reforçadores advindos do desempenho operante destacado no estudo.

Algumas das questões levantadas sobre o estudo de Banaco et al. (2004) foram consideradas na construção do delineamento da pesquisa de Regis Neto (2009). Nesse trabalho, muitos aspectos de programação foram cuidados. Primeiramente, utilizou-se para a base do experimento um responder sobre o qual se tivesse um controle maior: clicar submetido a esquema de reforçamento em intervalo variável. Para isso, um programa foi especialmente elaborado ${ }^{3}$ de forma que sete participantes, funcionários administrativos de uma universidade, pudessem clicar sobre um espaço na tela sobre o qual um círculo se movimentava em uma trajetória aleatória. Assim, o participante deveria "seguir" o círculo e clicar sobre ele para obter pontos de desempenho, como se fosse um jogo. Esses pontos eram contabilizados em um contador localizado acima da área de jogo. Acima e à direita da tela, outro contador registrava pontos recebidos em FT $1 s$ (isto é, independente que qualquer resposta específica do participante).

Os pontos atribuídos ao desempenho eram pagos em dinheiro e os pontos obtidos pela participação eram trocados por números para o concurso em uma loteria na qual concorriam somente os participantes do experimento, cujo prêmio também era dinheiro. Depois de estabilizada a taxa de respostas em VI ou 4 sessões de 15 minutos de submissão ao esquema, os participantes foram submetidos a 3 sessões de pareamento entre um tom (de $400 \mathrm{ciclos} / \mathrm{s}$ ) e um conjunto de estímulos aversivos:

3 O software foi elaborado por Thomas Woelz. o rosto de uma pessoa rindo e apontando para o participante com o dedo, um som de risada de escárnio e a perda de metade dos pontos pela participação (recebidos em FT 1s), com a consequente perda de escolha de números para a participação da loteria. Assim, buscou-se garantir que os pontos obtidos pelo desempenho não seriam afetados pela retirada de reforçadores utilizada como estimulação aversiva, à semelhança dos estudos com não humanos nos quais a estimulação aversiva não interfere na quantidade de reforçadores produzidos pelo desempenho dos sujeitos.

Os participantes do estudo de Regis Neto (2009) foram submetidos a duas sessões nas quais o intervalo de apresentação do CS durou 15 segundos. Em seguida, por mais uma sessão, para três participantes esse valor passou a ser de 30 segundos, enquanto para outros quatro participantes o valor foi modificado para 7,5 segundos. $\mathrm{Na}$ fase final do experimento, todos os participantes foram submetidos à extinção respondente.

Os resultados demonstraram que três participantes não apresentaram supressão no valor de 15 segundos de CS (a taxa de supressão variou entre 1,2 e 0,8 ), ao passo que os outros quatro participantes chegaram a apresentar taxa de supressão de até 0,05 - sendo que um deles apresentou uma alta taxa de supressão nas primeiras apresentações de CS-estimulação aversiva, deixando de apresentá-la durante os pareamentos subsequentes, e outros três apresentaram taxas de supressão gradativas à medida que os pareamentos CS-estimulação aversiva foram acontecendo.

Com os valores menores de duração do CS, todos os participantes que já haviam apresentado altas taxas de supressão na fase anterior continuaram a apresentá-la nesta nova fase. O participante desse grupo que não havia apresentado supressão com CS de 15 segundos de duração também não a apresentou nesta fase.

O mesmo ocorreu no grupo que teve o valor do CS aumentado para 30 segundos. Dois dos participantes desse grupo não haviam apresentado supressão na fase anterior e não apresentaram também nesta fase (dois deles, pelo contrário, apresentaram o que poderia ser chamado de indução: 1,5 e 1,97 vezes mais respostas durante o CS do que em períodos fora do CS). O terceiro participante, que já ha- 
via mostrado supressão na fase anterior, apresentou supressão também com esse novo valor de CS, mas numa única das apresentações do CS chegou a dobrar o número de respostas em relação ao período sem CS imediatamente anterior.

Os dados indicariam uma tendência maior de interferência (supressão ou indução) do pareamento sobre as respostas nas condições de CS mais curto e altas induções e menores supressões em condições de CS mais longo. Devido a algumas características de programação, a quantidade de reforços produzidos pelos participantes sofreu significativa variação. Essa variação criou um outro elemento de análise, ainda sob investigação. Uma correlação entre a frequência de reforço e a taxa de supressão pareceu corresponder aos dados da literatura, sem que as supressões observadas tenham ocorrido com os participantes com menor frequência de reforçamento na fase de fortalecimento (quatro sessões iniciais).

Em estudo subsequente de Regis Neto et al. (2009), seis participantes foram submetidos a um delineamento semelhante ao de Regis Neto (2009), com quatro sessões de 15 minutos de submissão a um esquema de VI 60s, o qual foi aperfeiçoado nessa ocasião, permitindo maior estabilidade no recebimento de reforços por sessão para todos os participantes. A fase experimental contava também com três sessões, a exemplo do trabalho anterior, mas agora com a duração do CS de 15 segundos em todas elas. Neste trabalho foram manipulados os eventos perturbadores (aversivos) subsequentes ao tom (CS).

Foram criadas três condições nas quais os participantes foram igualmente distribuídos: (a) condição na qual estavam presentes todos os eventos perturbadores (i.e., perda de pontos, som de risada e a imagem da pessoa rindo), (b) condição em que apenas a risada e a imagem eram apresentadas ao fim do CS e (c) uma terceira condição na qual apenas a perda de pontos ocorria ao fim do CS.

Os resultados indicaram que na Condição C ocorreram pequenas supressões e algumas induções de respostas bastante evidentes (números de respostas 2,0 e 1,76 maiores durante CS em um dos participantes). Na Condição B, nenhum dos participantes apresentou diminuição ou aumento significativo das respostas durante o CS. Já na Condição
A, não se observaram induções e para um dos participantes notou-se supressão de respostas em 1/3 das apresentações do CS.

Obviamente, ambas as pesquisas exigem replicações, modificações e adaptações, mesmo assim surpreendem os dados que indicam que a soma dos eventos ao fim do CS possa produzir um efeito diferente do resultante das apresentações destes individualmente. Isto é, a perda de pontos isolada tende a produzir indução de respostas; apenas a imagem com o som da risada não produz efeitos significativos e a apresentação conjunta destes produz alguma supressão nas respostas.

\section{Interações operantes na supressão condicionada}

Todos os resultados apresentados até aqui indicam que o estudo da supressão condicionada enquanto um modelo de ansiedade ainda requer mais pesquisas que visem a uma melhor descrição, com o desenvolvimento de métodos, a escolha de variáveis, de medidas e de parâmetros, tanto quando realizado com sujeitos humanos ou não humanos.

Há ainda outros relatos intrigantes na literatura que não foram explorados. Por exemplo, Sidman (1958) demonstrou que outras interações podem afetar o fenômeno da supressão condicionada. Já havia sido mostrado que, quando o operante em observação era uma resposta que pospunha a apresentação de um choque elétrico, sobreposições do pareamento som-choque elétrico inescapável produziam indução da resposta mantida pela esquiva. Portanto, era esperado que em uma situação complexa, na qual macacos foram expostos a um esquema concorrente "esquiva FI 20s - reforçamento por alimento em VI 4m", a sobreposição de um pareamento "som-choque elétrico inescapável" produzisse supressão no operante mantido por alimento e indução no operante que adiava a liberação do aversivo. Os resultados mostraram que tais respostas seguiram a mesma tendência: ambas tiveram indução durante o CS.

Sidman procurou nesse estudo muitas interações entre as variáveis estudadas, como a topografia das respostas que eram idênticas no primeiro experimento e passaram a ser bem distintas em experimentos posteriores, sendo inclusive afastadas no espaço da caixa experimental. Nenhuma des- 
sas manipulações produziu supressão no operante mantido por alimento durante o CS. Em outra manipulação, Sidman demonstrou que uma cadeia estabelecida entre as respostas de esquiva e apetitiva era o resultado produzido pelo controle aversivo do procedimento de supressão condicionada, tendo como subproduto a inesperada indução sobre o responder para a produção de estímulo apetitivo. Tal cadeia só pôde ser quebrada quando a produção do alimento foi modificada para razão fixa.

Interações desse tipo também devem ser mais bem estudadas, dada a relevância deste tipo de fenômeno para o dia a dia de humanos. Mas poucos são os trabalhos que exploraram as possíveis transposições desses achados para a vida cotidiana. Uma dessas tentativas pode ser encontrada em Bisaccioni (2008), que buscou apresentar interpretações de eventos cotidianos e algumas das implicações clínicas a partir dos estudos sobre supressão condicionada.

Tal linha de pesquisa pode esclarecer fenômenos importantes que ocorrem no contexto de aplicação da análise do comportamento, seja em âmbito clínico, hospitalar, escolar, organizacional, etc. Podemos tomar como exercício uma situação limite entre os dois primeiros contextos. Por exemplo, um cliente que acaba de receber o diagnóstico de um tumor maligno e que, ao invés de apresentar supressão condicionada, passa a trabalhar incessantemente - o que seria "inesperado".

\section{Considerações finais}

A despeito de o primeiro estudo nesta linha de pesquisa datar do início da década de 1940 (Estes \& Skinner, 1941) e de se encontrarem duas pesquisas da área no primeiro volume do $\mathrm{JEAB}^{4}$ (Sidman, 1958; Stein, Sidman, \& Brady, 1958), e embora possam ser identificadas pesquisas publicadas sobre supressão condicionada, muitas das questões ainda se encontram sem respostas, principalmente se forem consideradas as pesquisas com participantes humanos. Estas, em especial, necessitam de diversos aprimoramentos e desenvolvimento de tecnologias de pesquisa que constituam condições experimentais mais adequadas para o controle de variáveis e o registro de respostas. As pesquisas com humanos

4 Journal of the Experimental Analysis of Behavior. poderiam se beneficiar da criação de novas condições de coleta e de infraestrutura que envolvesse outros tipos de respostas.

Os dados de participantes humanos apresentados anteriormente apontam para a necessidade de algumas replicações e manipulações já realizadas com sujeitos não humanos, tais como: variações mais amplas na duração do estímulo condicional (e.g., Stein et al., 1958); diferentes frequências e esquemas de reforçamento (e.g., Lyon, 1963; Lyon, 1964; Lyon \& Felton, 1966); a utilização de novos estímulos no pareamento e a manipulação quanto à extensão dos efeitos do CS para outros estímulos com dimensão semelhante (e.g., Hoffman \& Fleshler, 1961, 1964; Hoffman, Selekman, \& Fleshler, 1966; Ray \& Stein, 1959).

Diante do apresentado, espera-se que esse artigo tenha, entre outras funções, a de evocar nos leitores comportamentos de engajamento nesta linha de pesquisa.

\section{Referências}

Banaco, R. A., Borges, N. B., Nogara, T., Oliveira, D., Rocha, A. C., Marangoni, A. M. . . . Mello, M. E. (2004). Produção de supressão condicionada em humanos: Um estudo inicial. Behaviors, 8, 13-16. Bisaccioni, P. (2008). Supressão condicionada: Contribuições da pesquisa básica para a prática clínica (Monografia de conclusão de curso). Núcleo Paradigma de Análise do Comportamento, São Paulo.

Blackman, D. (1968a). Conditioned suppression or acceleration as function of the behavioral baseline. Journal of the Experimental Analysis of Behavior, 11, 53-61.

Blackman, D. (1968b). Response rate, reinforcement frequency and conditioned suppression. Journal of the Experimental Analysis of Behavior, 11, 503-516.

Blackman, D. (1977). Conditioned suppression and the effects of classical conditioning on operant behavior. Em W. K. Honing \& J. E. R. Staddon (Orgs.), Handbook of operant behavior (pp. 340363). Englewood Cliffs: Prentice-Hall.

Calton, P. L., \& Didamo, P. (1960). Some notes on the control of conditioned suppression. Journal of the Experimental Analysis of Behavior, 3, 255-258. 
Costa, C. E. (2004) A natureza do reforçador como uma variável moduladora dos efeitos da história de reforço sobre o comportamento de seres humanos (Tese de doutorado). Universidade de São Paulo, São Paulo.

Estes, W. K., \& Skinner, B. F. (1941). Some quantitative properties of anxiety. Journal of Experimental Psychology, 29, 390-400.

Geller, I. (1960). The acquisition and extinction of conditioned suppression as a function of the base-line reinforcement. Journal of the Experimental Analysis of Behavior, 3, 235-240.

Hoffman, H. S., \& Fleshler, M. (1961). Stimulus factors in aversive controls: The generalization of conditioned suppression. Journal of the Experimental Analysis of Behavior, 4, 371-378.

Hoffman, H. S., \& Fleshler, M. (1964). Stimulus of aversive controls: Stimulus generalization of conditioned suppression following discrimination training. Journal of the Experimental Analysis of Behavior, 7, 233-239.

Hoffman, H. S., Selekman, W. L., \& Fleshler, M (1966). Stimulus factors in aversive controls: Conditioned suppression after equal training to two stimuli. Journal of the Experimental Analysis of Behavior, 9, 649-653.

Leitenberg, H. (1966). Conditioned acceleration and conditioned suppression in pigeons. Journal of the Experimental Analysis of Behavior, 9, 205-212.

Leslie, J. C. (1977). Effects of food deprivation and reinforcement magnitude on conditioned suppression. Journal of the Experimental Analysis of Behavior, 28, 107-115.

Leslie, J. C., Tierney, K. J., Robinson, C. P., Keenan, M., \& Watt, A. (1993). Differences between clinically anxious and non-anxious subjects in a stimulus equivalence training task involving threat words. The Psychological Record, 43, 153-161.

Lyon, D. (1963). Frequency of reinforcement as a parameter of conditioned suppression. Journal of the Experimental Analysis of Behavior, 6, 95-98.

Lyon, D. (1964). Some notes on conditioned suppression and reinforcement schedules. Journal of the Experimental Analysis of Behavior, 7, 289-191.

Lyon, D., \& Felton, M. (1966). Conditioned suppression and variable ratio reinforcement. Journal of the Experimental Analysis of Behavior, 9, 245-248.
Pliskoff, S.S. (1961). Rate-change effects during a pre-schedule-change stimulus. Journal of the Experimental Analysis of Behavior, 4, 383-386.

Ray, O. S., \& Stein, L. (1959). Generalization of conditioned suppression. Journal of the Experimental Analysis of Behavior, 2, 357-361.

Regis Neto, D. M. (2009). O efeito de diferentes durações do estímulo condicional na supressão condicionada em humanos (Dissertação de mestrado). Pontifícia Universidade Católica de São Paulo, São Paulo.

Regis Neto, D. M., Banaco, R. A., Bast, D., Bellodi, A. C., Bernardes, L. A., Bernardo, A. J. . . . Wang, M. A. L. (2009). Supressão condicionada em humanos: um estudo sobre os efeitos isolados e combinados de estímulos perturbadores. Behaviors, 13, 5-15

Sidman, M. (1958). By-products of aversive control. Journal of the Experimental Analysis of Behavior, 1, 265-280.

Stein, L., Sidman, M., \& Brady, J. V. (1958). Some effects of two temporal variables on conditioned suppression. Journal of the Experimental Analysis of Behavior, 1, 153-162.

Skinner, B. F. (2000). Ciência e comportamento humano (10a ed.; J. C. Todorov \& R. Azzi, Trads.). São Paulo: Martins Fontes. (Trabalho original publicado em 1953).

Tomanari, G. Y., Carvalho, A. A., Góes, Z. S., Lira, S. B., \& Viana, A. C. V. (2007). Pesquisando ao ensinar: Prática no laboratório didático analisa o comportamento verbal sob contingências de reforçamento positivo e negativo. Estudos de Psicologia, 24, 205-214.

Watt, A., Keenan, M., Barnes, D., \& Cairns, E. (1991). Social categorization and stimulus equivalence. The Psychological Record, 41, 33-50.

Watson, J. B. (1924). Behaviorism. New York: Norton.

\section{Informações do artigo}

\section{História do artigo}

Data de submissão em: 17/07/2010

Primeira decisão editorial em: 09/03/2011

Aceito para publicação em: 10/03/2011 